Bull. Austral. Math. Soc.

$35 \mathrm{~K} 22,35 \mathrm{~B} 05$

VoL. 52 (1995) [287-296]

\title{
CURVATURE EVOLUTION OF PLANE CURVES WITH PRESCRIBED OPENING ANGLE
}

\author{
NAOYUKI ISHIMURA
}

\begin{abstract}
We discuss the evolution of plane curves which are described by entire graphs with prescribed opening angle. We show that a solution converges to the unique self-similar solution with the same asymptotics.
\end{abstract}

\section{INTRODUCTION}

In [4], Ecker and Huisken discussed the mean curvature evolution of entire graphs with linear growth in $\mathbb{R}^{n+1}, n>2$. They proved the long time existence and under further assumptions, the convergence to a self-similar solution. In this note, on the other hand, we deal with the evolution of entire plane curves with prescribed opening angle. We consider the problem:

$$
\begin{gathered}
u_{t}=\frac{u_{x x}}{1+u_{x}^{2}}, \quad u>0 \text { in }-\infty<x<\infty, \quad t>0, \\
u_{x} \rightarrow-K_{2} \text { as } x \rightarrow-\infty \text { and } u_{x} \rightarrow K_{1}, \text { as } x \rightarrow \infty \text { for } t>0, \\
u(0, x)=u_{0}(x) .
\end{gathered}
$$

Here $0<K_{1} \leqslant K_{2}<\infty$ are prescribed constants. $u_{0}(x)$ is a given convex function assumed to satisfy (2) and

$$
\left(u_{0}-x u_{0 x}\right)^{2} \leqslant C\left(1+x^{2}\right)^{1-\delta}
$$

for some constants $C>0$ and $0<\delta \leqslant 1$. Notice that (4) does not follow from the convexity. In fact, there exits a convex function $u$ which satisfies (2) but where the growth of $\left(u-x u_{x}\right)^{2}$ dominates $x^{2(1-\delta)}$ for any $0<\delta \leqslant 1$. For example, we may take

Received 6th December, 1994

Results obtained while the author was visiting the Centre for Mathematics and its Applications, the Australian National University at Canberra, ACT 0200 during the period 1994/95. The support and friendly atmosphere of the institute is gratefully acknowledged. The author also expresses his sincere gratitude to Professor N.S. Trudinger and Professor H.-J. Kuo for warm encouragement during the stay. The work was partially supported by Grant-in-Aid for Scientific Research (No. 06740098), Japan Ministry of Education, Science and Culture.

Copyright Clearance Centre, Inc. Serial-fee code: 0004-9729/95 \$A2.00+0.00. 
$u=x-x(\log x)^{-1}$ for $x>e^{2}, u=2^{-1} e^{2}$ for $x \leqslant e^{2}$. The assumption of convexity will turn out not to be restrictive.

The growth condition (4) is essentially the same as (3) in Ecker and Huisken [4]. Thus the long time existence and the convergence to a self-similar solution will be established in a similar way. The novelty of our research consists in analysing a structure of self-similar solutions for (1).

To derive the self-similar equation corresponding to (1), we employ the so called similarity change of variables. We set

$$
u(t, x)=\sqrt{2(1+t)} \cdot U(\tau, y), \quad y=\frac{1}{\sqrt{2(1+t)}} x \quad \text { and } \quad \tau=\frac{1}{2} \log (1+t) .
$$

Then (1) becomes

$$
U_{\tau}=\frac{U_{y y}}{1+U_{y}^{2}}-U+y U_{y} .
$$

A stationary solution to (5) is called a self-similar solution. We show in Section 3 that the self-similar solution satisfying condition (2) exists uniquely. We remark that (4) is fulfilled automatically in this case. To summarise, we shall prove:

THEOREM 1.1. There exists a solution of (1) - (3) for all $t>0$. Under (4), it converges as $t \rightarrow \infty$ to the unique self-similar solution with the same asymptotics (2).

Mean curvature evolution is now a huge field of research. We refer to, for instance, Angenent [2], Ecker [3], Ecker and Huisken [5], Gage and Hamilton [6], Gage and Li [7], Grayson [8], Huisken [9], [10], and the references there in. (5) is a self-expanding equation. As to self-shrinking, we refer to, for instance, Altschuler, Angenent and Giga [1] and the pioneering observations of Leray [12].

After completing this work, Dr G.H. Williams kindly informed us of a related result; Broadbridge [13] considered the equilibrium solution of (5) for $y>0$ under the conditions $U_{y}(0)=$ given constant and $U \rightarrow 0$ as $y \rightarrow \infty$. He gives the exact representation of such solutions. We greatly thank $\mathrm{Dr}$ G.H. Williams for his interest in this work.

\section{EXISTENCE AND CONVERGENCE OF SOLUTIONS}

We first investigate the existence and the convergence of a solution to (1)-(4). As we mentioned before, we may argue similarly to Ecker and Huisken [4]. We include the proof, however, for completeness.

First, we derive an a-priori gradient bound. Set $w:=\log v, v:=\sqrt{1+u_{x}^{2}}$ and compute

$$
\begin{aligned}
w_{t} & =v^{-2} u_{x} u_{x t}=v^{-4} u_{x}\left(u_{x x}-2 v^{-2} u_{x} u_{x x}^{2}\right) \\
& =v^{-2} w_{x x}-v^{-4} u_{x x}^{2} \leqslant v^{-2} w_{x x}
\end{aligned}
$$


By applying the maximum principle, we find that $v$ is bounded, independent of $t$.

Next, we deal with curvature estimates. Write $K=u_{t}^{2}=\left(u_{x x} /\left(1+u_{x}^{2}\right)\right)^{2}$ to obtain

$$
\begin{aligned}
K_{t} & =v^{-2} K_{x x}-2 v^{-2} u_{t x}^{2}-4 v^{-4} u_{x} u_{x x} u_{t} u_{t x} \\
& \leqslant v^{-2} K_{x x}-v^{-4} u_{x} u_{x x} K_{x} .
\end{aligned}
$$

A uniform bound for $K$ now follows.

As in Proposition 4 of Ecker and Huisken [4], we can also infer that for every integer $i \geqslant 0$ there exists a constant $C(i)$ independent of $t$ such that

$$
t^{i+1}\left|K^{(i)}\right|^{2} \leqslant C(i) \text { for } t \geqslant 0
$$

We may safely omit the details. We simply remark that we have by induction in particular that $K^{(i)} \rightarrow 0$ as $|x| \rightarrow \infty$.

The convexity is preserved along the evolution. To see this, set $q=u_{x x}$ and differentiate (1) twice with respect to $x$. We arrive at

$$
q_{t}=v^{-2} q_{x x}-6 v^{-4} u_{x} u_{x x} q_{x}-4 v^{-4}\left(1-u_{x}^{2}\right) u_{x x}^{2} q
$$

Since $q \geqslant 0$ at $t=0$, the maximum principle implies $q \geqslant 0$ for $t>0$. Recall that $q \rightarrow 0$ as $|x| \rightarrow \infty$.

Convexity and uniform gradient bounds force $u_{x}$ to converge as $|x| \rightarrow \infty$. In view of the curvature bound $u_{t x} \rightarrow 0$ as $|x| \rightarrow \infty$, we conclude that (2) will be satisfied.

This completes the proof of long time existence for (1) - (3).

Now we investigate the convergence of a solution $u$ as $t \rightarrow \infty$. To make the calculation transparent, we introduce some notation.

Let $T:=v^{-1}\left(1, u_{x}\right), N:=v^{-1}\left(-u_{x}, 1\right)$ and $k:=-v^{-3} u_{x x}$. Then we get $T_{x}=$ $-v k N, N_{x}=v k T$ and

$$
T_{t}=v^{-2} u_{x t} N, \quad N_{t}=-v^{-2} u_{x t} T
$$

The corresponding quantities for (5) are distinguished by a tilde. That is, $\tilde{T}=$ 
$\widetilde{v}^{-1}\left(1, U_{y}\right), \tilde{N}=\widetilde{v}^{-1}\left(-U_{y}, 1\right)$ with $\tilde{v}=\sqrt{1+U_{y}^{2}}=v$ and so on.

(1) means $u_{t}=-v k$; we deduce

$$
\begin{aligned}
\frac{\partial}{\partial t}\langle X, N\rangle & -v^{-2} \frac{\partial^{2}}{\partial x^{2}}\langle X, N\rangle \\
& =-k-v^{-2} u_{z t}\langle X, T\rangle-v^{-2} \frac{\partial}{\partial x}(v k\langle X, T\rangle) \\
& =k^{2}\langle X, N\rangle-2 k \\
\frac{\partial}{\partial t}\langle X, N\rangle^{2} & -v^{-2} \frac{\partial^{2}}{\partial x^{2}}\langle X, N\rangle^{2} \\
& =2 k^{2}\langle X, N\rangle^{2}-4 k\langle X, N\rangle-2 v^{-2}\left(\frac{\partial}{\partial x}\langle X, N\rangle\right)^{2} .
\end{aligned}
$$

Here $\langle\cdot, \cdot\rangle$ denotes the inner product in $\mathbb{R}^{2}$.

From this, we estimate the growth rate of $\langle\tilde{X}, \tilde{N}\rangle^{2}$ as follows. Define $f:=$ $\left(1+2 t+x^{2}+u^{2}\right)^{\delta-1}$ and compute

$$
f_{t}-v^{-2} f_{x x}=-(\delta-2)(\delta-1)^{-1} f^{-1} f_{x}^{2} .
$$

We then obtain

$$
\begin{aligned}
\frac{\partial}{\partial t}\left(\langle X, N\rangle^{2} f\right) & -v^{-2} \frac{\partial^{2}}{\partial x^{2}}\left(\langle X, N\rangle^{2} f\right) \\
& =2 k^{2}\langle X, N\rangle^{2} f-4 k\langle X, N\rangle f-2 v^{-2}\left(\frac{\partial}{\partial x}\langle X, N\rangle\right)^{2} f \\
& -4 v^{-2}\langle X, N\rangle \frac{\partial}{\partial x}\langle X, N\rangle f_{x}-(2-\delta)(1-\delta)^{-1} v^{-2} f^{-1} f_{x}^{2} \\
& \leqslant 2\left(2 k^{2}+1\right)\langle X, N\rangle^{2} f+1 \\
\langle X, N\rangle^{2} f & \leqslant\left(C_{1}+t\right) e^{c_{2} t}
\end{aligned}
$$

for some constants $C_{1}, C_{2}$ independent of $t$. This implies

$$
\langle\tilde{X}, \tilde{N}\rangle \leqslant C_{3} e^{2 \tau} e^{c_{2} e^{2 \tau}}\left(1+y^{2}+U^{2}\right)^{1-\delta} .
$$

Here $C_{3}$ is again a constant independent of $t$.

Now we wish to show $U_{\tau}^{2} \rightarrow 0$ as $\tau \rightarrow \infty$. As a first step, we compute its evolution.

$$
\begin{aligned}
\frac{\partial}{\partial \tau} U_{\tau}^{2} & =2 U_{\tau}\left(\tilde{v}^{-2} U_{\tau y y}+\tilde{v}^{-1}\left(\tilde{v}^{-1}\right)_{y} U_{\tau y}-U_{\tau}+y U_{\tau y}\right), \\
\frac{\partial^{2}}{\partial y^{2}} U_{\tau}^{2} & =2 U_{\tau y}^{2}+2 U_{\tau} U_{\tau y y} \\
\left(\frac{\partial}{\partial \tau}-\widetilde{v}^{-2} \frac{\partial^{2}}{\partial y^{2}}\right) U_{\tau}^{2} & =-2 U_{\tau}^{2}+\left(y+2 \widetilde{v}^{-1}\left(\widetilde{v}^{-1}\right)_{y}\right) \frac{\partial}{\partial y} U_{\tau}^{2}-2 \widetilde{v}^{-2} U_{\tau y}^{2} .
\end{aligned}
$$


Due to the lack of a bound for $U_{\tau}^{2}$ as $|y| \rightarrow \infty$, we need to introduce the function $g=\left(\alpha+y^{2}+U^{2}\right)^{e-1} e^{e r}$ for some constants $0<\varepsilon<\delta$ and $\alpha>1$. $\alpha$ will be determined later. We have

$$
\begin{gathered}
\left(\frac{\partial}{\partial \tau}-\tilde{v}^{-2} \frac{\partial^{2}}{\partial y^{2}}\right) g=\varepsilon g+y g_{y}-2(\varepsilon-1)\left(\alpha+y^{2}+U^{2}\right)^{e-2}\left(1+y^{2}+U^{2}\right) e^{e \tau} \\
-(2-\varepsilon)(1-\varepsilon)^{-1} \tilde{v}^{-2} g^{-1} g_{y}^{2} \\
=(2-\varepsilon) g+y g_{y}+2(1-\alpha)(1-\varepsilon)\left(\alpha+y^{2}+U^{2}\right)^{e-2} e^{\epsilon \tau} \\
-(2-\varepsilon)(1-\varepsilon)^{-1} \tilde{v}^{-2} g^{-1} g_{y}^{2}
\end{gathered}
$$

Thus we find

$$
\begin{aligned}
\left(\frac{\partial}{\partial \tau}-\tilde{v}^{-2} \frac{\partial^{2}}{\partial y^{2}}\right) U_{\tau}^{2} g=g & \left(\frac{\partial}{\partial \tau}-\tilde{v}^{-2} \frac{\partial^{2}}{\partial y^{2}}\right) U_{\tau}^{2}+U_{\tau}^{2}\left(\frac{\partial}{\partial \tau}-\tilde{v}^{-2} \frac{\partial^{2}}{\partial y^{2}}\right) g \\
& -2 \tilde{v}^{-2} g_{y}\left(U_{\tau}^{2}\right) y \\
=-\varepsilon & U_{\tau}^{2} g+\left(y+2 \widetilde{v}^{-1}\left(\widetilde{v}^{-1}\right)_{y}\right) \frac{\partial}{\partial y}\left(U_{\tau}^{2} g\right) \\
& +2(1-\alpha)(1-\varepsilon)\left(\alpha+y^{2}+U^{2}\right)^{\varepsilon-2} e^{\varepsilon \tau} U_{\tau}^{2} \\
& -\tilde{v}^{-1}\left(\tilde{v}^{-1}\right)_{y} g_{y} e^{\varepsilon \tau} U_{\tau}^{2} \\
& -4 \tilde{v}^{-2} U_{\tau} U_{\tau y} g_{y}-2 \tilde{v}^{-2} g U_{\tau y}^{2} \\
& -(2-\varepsilon)(1-\varepsilon)^{-1} \tilde{v}^{-2} g^{-1} g_{y}^{2} U_{\tau}^{2}
\end{aligned}
$$

By virtue of $(2-\varepsilon)(1-\varepsilon)^{-1}>2$, the sum of the last three terms is negative from the Schwartz inequality. Recalling the curvature bound $\left|\left(\widetilde{v}^{-1}\right)_{y}\right| \leqslant C$, we find

$$
\left|\tilde{v}^{-1}\left(\tilde{v}^{-1}\right)_{y} g_{y} e^{e \tau} U_{\tau}^{2}\right| \leqslant \frac{\varepsilon}{2} U_{\tau}^{2} g
$$

if we choose $\alpha$ sufficiently large. We then obtain

$$
\left(\frac{\partial}{\partial \tau}-\widetilde{v}^{-2} \frac{\partial^{2}}{\partial y^{2}}\right) U_{\tau}^{2} g \leqslant-\frac{\varepsilon}{2} U_{\tau}^{2} g+\left(y+\widetilde{v}^{-1}\left(\widetilde{v}^{-1}\right)_{y}\right) \frac{\partial}{\partial y}\left(U_{\tau}^{2} g\right) .
$$

Since (6) makes $U_{\tau}^{2} g \rightarrow 0$ as $|y| \rightarrow \infty$ for every $\tau>0$, the maximum principle implies that $U_{\tau}^{2} g \leqslant C$ independent of $\tau$, which means

$$
U_{\tau}^{2} \rightarrow 0 \text { as } \tau \rightarrow 0 \text { for all fixed } y \in \mathbb{R} \text {. }
$$

Combining this with Theorem 3.1 below, we finally conclude that the proof of Theorem 1.1 is finished. 


\section{Structure of SELF-SIMILAR SOLUtions}

This section is concerned with the structure of solutions for the equation

$$
\begin{cases}U_{y y} /\left(1+U_{y}^{2}\right)=U-y U_{y}, U>0 & \text { for }-\infty<y<\infty \\ U(y) \rightarrow \infty & \text { as }|y| \rightarrow \infty\end{cases}
$$

Every solution of (7) will be seen to become asymptotically linear as $|y| \rightarrow \infty$. Moreover we have the following

TheOREM 3.1. Let $0<K_{1} \leqslant K_{2}<\infty$ be arbitrarily given constants. Then there exists a unique solution $U(y)$ to $(7)$, which is necessarily convex, such that

$$
\begin{aligned}
& U(y)=-K_{2} y+o\left(\frac{1}{|y|}\right) \text { as } y \rightarrow-\infty \\
& U(y)=K_{1} y+o\left(\frac{1}{|y|}\right) \text { as } y \rightarrow+\infty
\end{aligned}
$$

The proof proceeds similarly to the author's previous work [11], but we carry it out here, since it is slightly different and the uniqueness is newly established.

Before going into details, we observe that it suffices to consider only the symmetric case $U_{y}(0)=0$. To see this, we argue as follows. Since the linear function $U=a y$ solves (7) for every $a \in \mathbb{R}, U(y)$ claimed in Theorem 3.1 is in the region $\left\{U>-K_{2} y\right\} \cap\{U>$ $\left.K_{1} y\right\}$ from an interior touching principle. Thus, (7) can be parametrised by polar coordinates; $(U(\theta), y(\theta)):=(R(\theta) \sin \theta, R(\theta) \cos \theta)$ with $R^{2}=U^{2}+y^{2}$ for $\theta_{1}<\theta<\theta_{2}$. $\theta_{i} \in(0, \pi)$ are determined by $\theta_{1}=\arctan K_{2}, \theta_{2}=-\arctan K_{1}$. (7) is equivalent to

$$
-k=\langle X, N\rangle
$$

where

$$
X:=(U(\theta), y(\theta)), N:=v^{-1}\left(-y_{\theta}, U_{\theta}\right), v^{2}:=U_{\theta}^{2}+y_{\theta}^{2}
$$

and

$$
k:=\frac{U_{\theta \theta} y_{\theta}-U_{\theta} y_{\theta \theta}}{v^{3}}
$$

(7) is now transformed into

$$
\begin{cases}1=\left(R R_{\theta \theta}-2 R_{\theta}^{2}-R^{2}\right) /\left(R^{2}+R_{\theta}^{2}\right)^{2} & \text { for } \theta_{1}<\theta<\theta_{2}, \\ R \rightarrow \infty & \text { as } \theta \rightarrow \theta_{1}, \theta_{2} .\end{cases}
$$

One sees that (8) is invariant under the translation $\theta \mapsto \theta+c, c \in \mathbb{R}$; the solution of (8) is characterised through $\theta_{2}-\theta_{1}$. Only the centre of scaling is different. Therefore, we just have to establish the next proposition which says that for every $\theta_{2}-\theta_{1} \in(0, \pi)$, there corresponds the symmetric solution uniquely. 
Proposition 3.2. There exists a one-parameter family of convex solutions $U\left(y ; U_{0}\right)$ for $(7)$ such that

$$
U_{y}(0)=0, \quad U(0)=U_{0}>0 .
$$

We define $\alpha\left(U_{0}\right):=\lim _{y \rightarrow \infty} U_{y}\left(y ; U_{0}\right)$. The function $U_{0} \mapsto \alpha\left(U_{0}\right)$ is strictly increasing and provides a homeomorphism from $\mathbb{R}^{+}=\{x>0\}$ into itself.

Proof: We divide the proof into several steps.

1. $U\left(y ; U_{0}\right)$ exists for all $y \in \mathbb{R}$.

The solution $U\left(y ; U_{0}\right)$ of $(7)$ with $U(0)=U_{0}$ and $U_{y}(0)=0$ certainly exists on some interval $\left(-y_{1}, y_{1}\right) . U_{y y}>0$ by virtue of $U_{y y}(0)=U_{0}>0$. If there exists a point $y_{2}>0$ such that $U_{y y}\left(y_{2}\right)=0$ but $U, U_{y}$ are bounded, then we have $U^{(n)}\left(y_{2}\right)=0$ for $n \geqslant 2$, performing successive differentiations on (7). The analyticity of the regular elliptic equation leads to

$$
U\left(y ; U_{0}\right)=U_{y}\left(y_{2}\right)\left(y-y_{2}\right)+U\left(y_{2}\right)
$$

a contradiction.

If there exists a point $y_{3}>0$ with $U\left(y_{3}\right)=\infty$ or $U_{y}\left(y_{3}\right)=\infty$, then we may choose $C_{1}$ so that $U=C_{1} y$ touches $U\left(y ; U_{0}\right) ; U\left(y_{;} U_{0}\right) \geqslant C_{1} y$ on $\left(0, y_{3}\right)$ and $U\left(y_{4} ; U_{0}\right)=$ $C_{1} y_{4}, U_{y}\left(y_{4} ; U_{0}\right)=C_{1}$ for some $y_{4} \in\left(0, y_{3}\right) . U=C_{1} y$ is a solution to (7). Thus, this cannot happen; $U\left(y ; U_{0}\right)$ exists on all $y \in \mathbb{R}$, keeping the convexity.

2. $\alpha\left(U_{0}\right):=\lim _{y \rightarrow \infty} U_{y}\left(y ; U_{0}\right)<\infty$ is defined.

Let $I\left(y ; U_{0}\right):=\arctan U_{y}\left(y ; U_{0}\right)$. Since $I(0)=0, I_{y}(y)>0$, we infer that $0<$ $I(y)<\pi / 2$ for $y>0$ and the limit $\beta\left(U_{0}\right):=\lim _{y \rightarrow \infty} I\left(y ; U_{0}\right) \leqslant \pi / 2$ exists. We show that $\beta\left(U_{0}\right)<\pi / 2$. If $\beta\left(U_{0}\right)=\pi / 2$, then $U_{y}$ increases to $\infty$ as $y \rightarrow \infty$. However, the concavity of $\arctan x$ for $x>0$ implies $I_{y}(y) \rightarrow 0$ as $y \rightarrow \infty$; that is, $U-y U_{y} \rightarrow 0$. Therefore, $U / y-U_{y}=0(1 / y), U_{y y}=0\left(1 / y^{2}\right)$, which is a contradiction to $U_{y} \rightarrow \infty$ as $y \rightarrow \infty$. The claim is proved.

To proceed further, we need a lemma.

Lemma 3.3. Every distinct pair of solutions to (7) intersect at most once.

Proof: For a contradiction, suppose two distinct solutions $U_{1}(y), U_{2}(y)$ intersect at $y_{1}<y_{2}$ with $U_{1}(y)>U_{2}(y)$ for $y_{1}<y<y_{2}$. Integration of (7) over the interval 
$\left(y_{1}, y_{2}\right)$ gives

$$
\begin{aligned}
\arctan U_{1 y}\left(y_{2}\right) & -\arctan U_{1 y}\left(y_{1}\right)=\int_{y_{1}}^{y_{2}}\left(U_{1}-y U_{1 y}\right) d y \\
& =\int_{y_{1}}^{y_{2}} 2 U_{1} d y-y_{2} U_{1}\left(y_{2}\right)+y_{1} U_{1}\left(y_{1}\right) \\
& >\int_{y_{1}}^{y_{2}} 2 U_{2} d y-y_{2} U_{2}\left(y_{2}\right)+y_{1} U_{2}\left(y_{1}\right) \\
& =\arctan U_{2 y}\left(y_{2}\right)-\arctan U_{2 y}\left(y_{1}\right) .
\end{aligned}
$$

We find a contradiction in view of $U_{1 y}\left(y_{1}\right) \geqslant U_{2 y}\left(y_{1}\right)$ and $U_{1 y}\left(y_{2}\right) \leqslant U_{2 y}\left(y_{2}\right)$.

3. $\alpha\left(U_{10}\right)<\alpha\left(U_{20}\right)$ for $U_{10}<U_{20}$.

Lemma 3.3 gives $\alpha\left(U_{10}\right) \leqslant \alpha\left(U_{20}\right)$. We show the strictness of the inequality indirectly. Suppose for some $U_{10}<U_{20}$ we have $\alpha\left(U_{10}\right)=\alpha\left(U_{20}\right)$. Consider $\bar{U}_{h}\left(y ; U_{0}\right):=U_{1}\left(y ; U_{10}\right)+h . \bar{U}_{h}$ is a supersolution, satisfying $\bar{U}_{h y}=U_{1 y}$ and $\bar{U}_{h}-y \bar{U}_{h y}>U_{1}-y U_{1 y}$. Since $\bar{U}_{h y y}(0)=U_{10}<U_{20}=U_{2 y y}(0)$, by letting $h \rightarrow 0$ we discover some $\bar{h}$ so that $\bar{U}_{\bar{h}}$ touches $U_{2}$ from above. This is prohibited by the interior touching principle.

4. $\lim _{U_{0} \rightarrow 0} \alpha\left(U_{0}\right)=0$.

If not, there is a $\delta>0$ such that

$$
\lim _{U_{0} \rightarrow 0} \alpha\left(U_{0}\right)=\delta
$$

Introduce a comparison function

$$
\bar{U}(y):=U_{0}+C-\sqrt{C^{2}-y^{2}} \quad \text { where } \quad C=\frac{1+\sqrt{1+\delta^{2}}}{\delta^{2}} U_{0} .
$$

$\bar{U}(y)$ has a tangency with $U=\delta y$ at $y_{1}=U_{0}\left(1+\sqrt{1+\delta^{2}}\right) / \delta \sqrt{1+\delta^{2}}<C$. That is

$$
\delta y_{1}=U_{0}+C-\sqrt{C^{2}-y^{2}} \text { and } \delta=\frac{y_{1}}{\sqrt{C^{2}-y_{1}^{2}}}
$$

Since $\bar{U}(y)=U\left(0 ; U_{0}\right)=U_{0}, \bar{U}_{y}(0)=U_{y}\left(0 ; U_{0}\right)=0$ and

$$
\bar{U}_{y y}(0)=C^{-1}>U_{y y}\left(0 ; U_{0}\right)=U_{0}
$$

we see that $\bar{U}(y)>U\left(y ; U_{0}\right)$ in some neighbourhood of $y=0$, taking $U_{0}$ sufficiently small. Furthermore, we compute

$$
\frac{\bar{U}_{y y}}{1+\bar{U}_{y}^{2}}-\bar{U}+y \bar{U}_{y}=\frac{1}{\sqrt{C^{2}-y^{2}}}\left\{1-\left(U_{0}+C\right) \sqrt{C^{2}-y^{2}}+C^{2}\right\}>0
$$


on $0<y<y_{1}+\varepsilon<C$ for some $\varepsilon>0$, choosing $U_{0}$ smaller if necessary. Recalling that $U\left(y ; U_{0}\right)$ cannot intersect with $U=\delta y$ and so $U\left(y_{1} ; U_{0}\right)>\delta y_{1}$, we conclude this is impossible.

5. $\lim _{U_{0} \rightarrow \infty} \alpha\left(U_{0}\right)=\infty$.

Suppose on the contrary that $\lim _{U_{0} \rightarrow \infty} \alpha\left(U_{0}\right)=M<\infty$. We deduce that for any $\varepsilon>0$, there exists $y_{1}>0$ and $U_{10}$ such that

$$
0<U\left(y ; U_{0}\right)-M y<\varepsilon \text { for } y \geqslant y_{1} \text { and } U_{0}>U_{10} \text {, }
$$

invoking $U-y U_{y} \rightarrow 0$ as $y \rightarrow \infty$. Define $\bar{U}(y):=U\left(y ; U_{10}\right)+2 \varepsilon$ and compare this with the solution $U\left(y ; U_{10}+3 \varepsilon\right)$. We have $\bar{U}(0)<U\left(0 ; U_{10}+3 \varepsilon\right)$ and $\left.\bar{U}\left(y_{1}\right)>M y_{1}+2 \varepsilon\right\rangle$ $U\left(y_{1} ; U_{10}+3 \varepsilon\right) . \bar{U}$ is a supersolution and hence, this is a contradiction.

6. $U_{0} \mapsto \alpha\left(U_{0}\right)$ is continuous.

By virtue of the monotonicity of $\alpha\left(U_{0}\right)$, the set of discontinuity points $\left\{U_{i 0}\right\}$ is countable. Let $U_{10}$ be one of them. For some $\varepsilon>0$, we find

$$
\lim _{U_{0} \uparrow U_{10}} \alpha\left(U_{0}\right)+\varepsilon \leqslant \lim _{U_{0} \downarrow U_{10}} \alpha\left(U_{0}\right) .
$$

From the convexity of $U$, there exists $y_{1}$, such that

$$
\lim _{U_{0} \uparrow U_{10}} U_{y}\left(y ; U_{0}\right)+\frac{\varepsilon}{2} \leqslant \lim _{U_{0} \downarrow U_{10}} U_{y}\left(y ; U_{0}\right) \text { for all } y \geqslant y_{1} .
$$

The continuity with respect to the initial value on the bounded interval $\left[0, y_{1}\right]$ does not allow this.

The proof is now complete.

A usual limit process shows that the case $K_{1}=0$ is valid in Theorem 3.1. To be precise, we obtain

Corollary 3.4. For every $K>0$, there exists the unique solution $U(y)$ of (7) such that

$$
\begin{aligned}
& U(y)=-K y+o\left(\frac{1}{|y|}\right) \text { as } y \rightarrow-\infty \\
& U(y)=o\left(\frac{1}{|y|}\right) \text { as } y \rightarrow+\infty
\end{aligned}
$$

Proof: Let $\left\{L_{n}\right\}, n=1,2, \ldots$, be a sequence with $K>L_{1}>L_{2}>\cdots \rightarrow 0$. Consider the corresponding sequence of solutions $\left\{U_{n}(y)\right\}$ to (7), which are given by $K_{2}=K$ and $K_{1}=L_{n}$ in Theorem 3.1. $U\left(y ; U_{0}\right)$ with $\alpha\left(U_{0}\right)>K$ for $y \in \mathbb{R}$ and $U=-K / 2 y$ for $y<0$ serve as upper and lower barriers on any bounded interval, respectively. Moreover, we have $0<U_{n y}(y)<K$. We can extract a convergent subsequence on any bounded interval. A standard diagonal argument yields the desired conclusion. Finally, the uniqueness follows as before; transform the equation into (8), translate the $\theta$ variable and appeal to the uniqueness of the symmetric solutions. 


\section{REFERENCES}

[1] S.J. Altschuler, S.B. Angenent and Y. Giga, 'Mean curvature flow through singularities for surfaces of rotation', Hokkaido Univ. Preprint Series 130 (1991).

[2] S.B. Angenent, 'On the formation of singularities in the curve shortening flow', J. Differential Geom. 33 (1991), 601-633.

[3] K. Ecker, 'On mean curvature flow of spacelike hypersurfaces in asymptotically flat space times', J. Austral. Math. Soc. Ser. A 55 (1993), 41-59.

[4] K. Ecker and G. Huisken, 'Mean curvature evolution of entire graphs', Ann. of Math. 130 (1989), 453-471.

[5] K. Ecker and G. Huisken, 'Interior estimates for hypersurfaces moving by mean curvature', Invent. Math. 105 (1991), 547-569.

[6] M.E. Gage and R.S. Hamilton, 'The shrinking of convex plane curves by the heat equation', J. Differential Geom. 23 (1986), 69-96.

[7] M.E. Gage and Y. Li, 'Evolving plane curves by curvature in relative geometries II', Duke Math. J. 75 (1994), 79-98.

[8] M.A. Grayson, 'The heat equation shrinks embedded plane curves to round points', $J$. Differential Geom. 26 (1987), 285-314.

[9] G. Huisken, 'Flow by mean curvature of convex surfaces into spheres', J. Differential Geom. 20 (1984), 237-266.

[10] G. Huisken, 'Non-parametric mean curvature evolution with boundary conditions', $J$. Differential Equations 77 (1989), 369-378.

[11] N. Ishimura, 'Self-similar solutions for the Gauss curvature evolution of rotationally symmetric surfaces', (submitted 1994).

[12] J. Leray, 'Sur le movement d'un liquid visqueux emplissant l'espace', Acta Math. 63 (1934), 193-248.

[13] P. Broadbridge, 'Exact solvability of the Mullins nonlinear diffusion model of groove development', J. Math. Phys. 30 (1989), 1648-1651.

Department of Mathematics

University of Tokyo

Hongo, Tokyo 113

Japan 